\title{
The Socio-Political Perspectives of Neuroethics: An Approach to Combat the Reproducibility Crisis in Science?
}

\section{Emily Doerksen \& Jean-Christophe Boivin}

To cite this article: Emily Doerksen \& Jean-Christophe Boivin (2022) The Socio-Political Perspectives of Neuroethics: An Approach to Combat the Reproducibility Crisis in Science?, AJOB Neuroscience, 13:1, 31-32, DOI: 10.1080/21507740.2021.2001092

To link to this article: https://doi.org/10.1080/21507740.2021.2001092

曲 Published online: 21 Dec 2021.

Submit your article to this journal $\widetilde{ }$

Џ Article views: 28

Q View related articles $\longleftarrow$

View Crossmark data $\asymp$ 


\title{
The Socio-Political Perspectives of Neuroethics: An Approach to Combat the Reproducibility Crisis in Science?
}

\author{
Emily Doerksen $^{\mathrm{a}}$ (D) and Jean-Christophe Boivin ${ }^{\mathrm{a}}$ (I)
}

McGill University

In their article, Dubljević, Trettenbach, and Ranisch (2022) make a compelling case for incorporating a "socio-political perspective" when discussing the ethical underpinnings of neuroscience in addition to the preexisting knowledge-driven, technology-driven, and healthcare driven perspectives considered to accompany neuroethics as a discipline. The latter three perspectives represent established concerns and considerations in neuroethics; however, the authors note that they alone are insufficient to impose limits pertaining to the realworld implications stemming from neuroscience. Accordingly, a socio-political perspective in neuroethics plays a prescriptive role in neuroscience research by “... providing a meaningful platform for informed public discourse and reflection, which may enable and empower democratic deliberation and decision making..." (Dubljević, Trettenbach, and Ranisch 2022, 17). Informed by Rawls' proposed four roles of political philosophy, the authors argue that a socio-political perspective in neuroethics would serve the following roles: clarifying and resolving conflicts, orienting the public to the moral status of neurotechnology, reconciling the public with neurotechnological changes, and probing the limits of practical social and neurotechnological possibilities (Dubljević, Trettenbach, and Ranisch 2022). With these principles, neuroscience can avoid falling prey to "hype and hyperbole," which commonly afflicts neuroscience and its research outputs. The article notes that hype is an issue in neuroscience and other scientific disciplines in part because generating hype for science is integral to many institutions that support scientific inquiries (e.g., universities, journals, funding agencies). Here, we consider whether this same concern for hype is a primary factor contributing to the reproducibility crisis in science. We note that this search for 'hype-able' findings has contributed to the publish-or-perish culture in academia and the reinforcement of publication bias, or the "file drawer effect." Finally, we consider how a sociopolitical perspective at an institutional level could help mitigate the effects of hype across all fields of science.

The tendency to hype scientific results has had a tangible impact on many disciplines in addition to neuroscience. For example, hype in the media leads to a misrepresentation of how scientific findings are presented to the public. Further, the desire for hype-able research at an institutional level creates fertile ground for scientific malpractice, manifesting most clearly as the infamous reproducibility crisis in science. The reproducibility crisis is due to the realization that published studies and their findings are not replicable in many scientific fields (Baker 2016). Successful replication rates of previous study results vary from $10 \%$ to $40 \%$, depending on the field and study (Baker 2016). Authors have identified multiple questionable practices that contribute to the crisis. These include p-hacking-the manipulation of a dataset done to reach a specific desired statistical threshold; HARKing - the formulation or modification of a hypothesis to make it fit the obtained results; and cherrypicking - the presentation of incomplete evidence which consciously excludes data that may contradict a favored hypothesis (Frias-Navarro et al. 2020). In extreme cases, practices stemming from the crisis have manifested as the blatant falsification of results (Frias-Navarro et al. 2020). A consequence of such practices is that scientific research may offer a false picture of scientific realities, with non-reproducible results cited more frequently to support an idea or a theory than studies that could be successfully replicated (Serra-Garcia and Gneezy 2021). 
However, it should be noted that these practices are not simply due to ill-intentioned scientists. The publish-orperish culture in academia is a leading cause of these practices, whereby grants, funding, and promotions are given according to how impactful the conducted research is, or, in some cases, how much hype it generates (Begley and Ioannidis 2015; Fanelli 2010).

The pressure for scientists to produce hype-able findings creates another problem directly contributing to the reproducibility crisis: the file drawer effect. Coined by Rosenthal (1979), the file drawer effect is understood as a publication bias wherein negative study results are confined to a scientist's "file drawer" instead of seeing the light of publication. With the pressure to publish in the foreground, it becomes challenging for researchers to produce results appealing to funders and the public while accurately presenting ineffectual or unremarkable data (Fanelli 2010). Research tolerates a false positive rate of $5 \%$. As such, a published article very well may offer findings from an experiment that was successful once, but in reality, failed far more often than it succeeded. Since these "failures" never come to light, it is difficult to assess their impact and may force the scientific community to "groupthink." That is, it leads the scientific community to reach a false consensus about certain scientific findings and theories. The file drawer effect becomes even more pernicious when combined with the effects of the publish-or-perish culture, as negative results would be the most efficient way to expose such questionable research practices (Fanelli 2010).

Authors that comment on the reproducibility crisis often conclude that multiple institutional changes are required to reduce its effects, although there is no one clear solution to address these concerns. We suggest that incorporating the socio-political perspective as put forward by Dubljević and company within scientific institutions may help move science forward from the effects of the reproducibility crisis. Their socio-political perspective offers ethical guidance for policymakers and the public (Dubljević, Trettenbach, and Ranisch 2022). When it comes to the reproducibility crisis, a socio-political perspective could indeed serve to (1) clarify and resolve conflicts stemming from the reproducibility crisis; (2) orient the public to the moral status of irreproducible results; (3) reconcile the public with realistic scientific changes; and (4) probe the limits of practical social and scientific possibilities. The authors argue that a socio-political perspective guides us in ethical deliberation by keeping policies and regulations relevant to the application of new science in clear view. As a result, the socio-political approach aims to ensure that neuroethics stays its course by preventing exaggerated scientific hype in search of funding sources. The application of the socio-political approach for science at an institutional level, that is, funding agencies and universities, would mitigate scientists' concern for hype-able results. It also would ensure that institutions are committed to practices that offer reliable communication of all data within the scientific community to provide the most accurate portrait of scientific findings to the socio-political sphere. As such, a socio-political perspective would contribute to improving scientific practices by increasing transparency in research conduct and communication of findings. Such a commitment would encourage something similar to an "open science" approach, effectively creating an open forum where scientists may share data they obtained in various experiments regardless of the data's "significance." Ultimately, the incorporation of the socio-political perspective serves to ground scientific practice by reminding scientific institutions, policymakers, and scientists, of the discourse between science and society.

\section{FUNDING}

The author(s) reported there is no funding associated with the work featured in this article.

\section{ORCID}

Emily Doerksen (D) http://orcid.org/0000-0002-3595-3755

Jean-Christophe Boivin (iD http://orcid.org/0000-00017757-4625

\section{REFERENCES}

Baker, M. 2016. 1,500 scientists lift the lid on reproducibility. Nature 533 (7604):452-4. doi:10.1038/533452a.

Begley, C. G., and J. P. Ioannidis. 2015. Reproducibility in science: Improving the standard for basic and preclinical research. Circulation Research 116 (1):116-26. doi:10. 1161/CIRCRESAHA.114.303819.

Dubljević, V., K. Trettenbach, and R. Ranisch. 2022. The socio-political roles of neuroethics and the case of Klotho. AJOB Neuroscience 13 (1):10-22. doi:10.1080/ 21507740.2021.1896597.

Fanelli, D. 2010. "Positive" results increase down the hierarchy of the sciences. PLOS One 5:4. doi:10.1371/journal. pone.0010068.

Frias-Navarro, D., J. Pascual-Llobell, M. Pascual-Soler, J. Perezgonzalez, and J. Berrios-Riquelme. 2020. Replication crisis or an opportunity to improve scientific production? European Journal of Education 55 (4):618-31. doi:10. 1111/ejed.12417.

Rosenthal, R. 1979. The file drawer problem and tolerance for null results. Psychological Bulletin 86 (3):638-41. doi: 10.1037/0033-2909.86.3.638.

Serra-Garcia, M., and U. Gneezy. 2021. Nonreplicable publications are cited more than replicable ones. Science Advances. 7:21. doi:10.1126/sciadv.abd1705. 